\title{
CORELib: COsmic Ray Event LIBrary
}

\section{Simona Maria Stellacci}

INFN - Gruppo Collegato di Salerno

Via Giovanni Paolo II - Fisciano, Italy

E-mail: stellacciesa.infn.it

Bernardino Spisso

INFN - Sec. Napoli

Strada Comunale Cinthia, 80126 Napoli,Italy

E-mail: spissoena.infn.it

\section{Carmelo Pellegrino}

CNAF

Viale Carlo Berti Pichat, 6, 40127 Bologna, Italy

E-mail: carmelo.pellegrinodinfn.it

\section{Cristiano Bozza}

Università degli Studi di Salerno

Via Giovanni Paolo II - Fisciano, Italy

E-mail: cbozza@unisa.it

\section{Tommaso Chiarusi}

INFN - Sec. Bologna

Viale Carlo Berti Pichat- Bologna,Italy

E-mail:tommaso.chiarusi@bo.infn.it

\section{Rosa Coniglione, Emidio Giorgio}

LNS (Laboratori Nazionali del Sud)

Via S. Sofia, 62, 95125 Catania

E-mail:rosa.coniglione@Ins.infn.it,emidio.giorgio@infn.it

\section{Paolo Lo Re, Pasquale Migliozzi}

INFN - Sec. Napoli

Strada Comunale Cinthia, 80126 Napoli,Italy

E-mail:paolo.lore@na.infn.it,pasqualemigliozzi69@gmail.com

\section{Agnese Martini}

LNF (Laboratori Nazionali di Frascati

Via Enrico Fermi, 40- Frascati,Italy

E-mail:agnese.martini@lnf.infn.it

CORELib is a library of simulated events that is meant for open access to satisfy a broad range of needs. The physical parameters of CORELib production are set to achieve a general purpose high statistics production.

The New Era of Multi-Messenger Astrophysics - Asterics2019

25-29 March, 2019

Groningen, The Netherland

(C) Copyright owned by the author(s) under the terms of the Creative Commons

Attribution-NonCommercial-NoDerivatives 4.0 International License (CC BY-NC-ND 4.0). 


\section{INTRODUCTION}

Cosmic rays are a source of background for many astroparticle and astronomy experiments. The computing time needed to simulate air showers increases exponentially with the energy of the primary cosmic ray. The main focus of CORELib is to provide a collection of simulated showers induced by cosmic rays and a common framework to produce and manage such large production. Originally developed for the Research Infrastractures (ESFRI) in ASTERICS, especially KM3NeT, CORELib is potentially useful also outside of the astronomical community. For example, simulations of air showers induced by high energy primary cosmic rays are very interesting for muon radiography, which is employed both to study the inner structures of volcanoes and for archaeological investigations of big monuments such as pyramids.

The production is obtained by using the widely used CORSIKA-7.5000 as generator and the GRID infrastructure as computational resources. The KM3NeT's VO (Virtual Organization) has been used, mainly the computational and storage resources located in the Naples university.

\section{CORSIKA}

Initially developed to run simulations for the KASCADE experiment, CORSIKA (Cosmic Ray Simulation for KAscade) [1] is a detailed Monte Carlo program to follow step by step the evolution of extensive air showers (EAS) produced by the interaction of cosmic rays with the atmosphere. EAS induced by protons and light nuclei up to $\mathrm{Fe}$ or photons can be simulated. The interactions with air nuclei produce secondary particles that are tracked along their trajectories and their parameters are stored in files when a set observation level is reached. Each simulation run produces two types of output files:

- Control output file: a text file in which all the standard output of the corresponding run is stored, first of all the datacards of the event;

- Particle list: a compressed binary file that contains:

- all the information about the secondary particles;

- photons produced by the Cherenkov effect (if this option has been selected ).

\section{CORELib}

Each event collected in CORELib represents a different simulated shower. The showers produced are generated by primary cosmic rays with zenith angle between 0 and 89 degrees. The observation level is placed at sea level, while the Standard European Atmospheric Model is used. The selected parameters of simulation are available and easily accessible to the user, encoded in a CORSIKA datacard. The choice of the parameters has been done with the KM3NeT Simulation and Analysis Working Groups [2].

In order to work out the best selection in terms of computation time and quality of production, a "pilot production" of CORELib has been made. Only showers induced by protons were considered and the simulated energy spectrum has a power law with spectral index -2 .

The "full scale production", instead, consists of several kinds of primary cosmic rays: showers induced by protons and heavy nuclei ( $\mathrm{He}, \mathrm{C}, \mathrm{N}, \mathrm{O}, \mathrm{Fe}$ ) are simulated (Table 1).

For proton-induced showers, two productions are available: with and without Cherenkov radiation (Table 2).

Besides the energy spectrum tested in the pilot production, a flat energy spectrum (spectral index $=0$ ) to increase the statistics at high energy has been evaluated (Table 2).

The aim is to that enhance event samples with features that are worth studying. Both productions are made with different high energy hadronic interaction models: QGSJET01 with the CHARM option selected to enable the treatment of charmed states, QGSJETI and EPOS with the TAULEP option selected to enable the treatment of tau. GHEISHA has been chosen as a common low energy model. 
S.M.Stellacci, B.Spisso, C.Pellegrino, C.Bozza, T.Chiarusi, R.Coniglione, E.Giorgio, P.Lo Re, P.Migliozzi, A. Martini - CORELib

\begin{tabular}{|c|c|c|c|c|c|c|c|c|c|c|c|c|c|c|}
\hline \multicolumn{7}{|c|}{ Heavy nuclei (He-CNO-Fe) induced showers } & \multicolumn{8}{|c|}{ Proton induced showers } \\
\hline \multirow[t]{2}{*}{$\begin{array}{l}\text { High Energy } \\
\text { Model }\end{array}$} & \multirow[t]{2}{*}{$\begin{array}{l}\text { Low Energy } \\
\text { Model }\end{array}$} & \multicolumn{2}{|c|}{ Option } & \multicolumn{2}{|c|}{$\begin{array}{l}\text { Cherenkov } \\
\text { Radiation }\end{array}$} & \multirow{2}{*}{$\begin{array}{l}\text { STATUS } \\
\alpha=-2\end{array}$} & \multirow[t]{2}{*}{$\begin{array}{l}\text { High Energy } \\
\text { Model }\end{array}$} & \multirow[t]{2}{*}{$\begin{array}{l}\text { Low Energy } \\
\text { Model }\end{array}$} & \multicolumn{2}{|c|}{ Option } & \multicolumn{2}{|c|}{$\begin{array}{l}\text { Cherenkov } \\
\text { Radiation }\end{array}$} & \multicolumn{2}{|c|}{ STATUS } \\
\hline & & TAULEP & CHARM & with & without & & & & TAULEP & CHARM & with & without & $\alpha=-2$ & $\alpha=0$ \\
\hline QGSJET01 & GHEISHA & & $\mathrm{x}$ & & $\mathrm{x}$ & DONE & QGSJET01 & GHEISHA & & $\mathrm{x}$ & $\mathrm{x}$ & $\mathrm{x}$ & DONE & DONE \\
\hline QGSJET01 & GHEISHA & $\mathrm{x}$ & & & $\mathrm{x}$ & DONE & QGSJET01 & GHEISHA & $\mathrm{x}$ & & $\mathrm{x}$ & $\mathrm{x}$ & DONE & DONE \\
\hline QGSJET II-04 & GHEISHA & $\mathrm{x}$ & & & $\mathrm{x}$ & DONE & QGSJET II-04 & GHEISHA & $\mathrm{x}$ & & $\mathrm{x}$ & $\mathrm{x}$ & DONE & DONE \\
\hline EPOS & GHEISHA & $\mathrm{x}$ & & & $\mathrm{x}$ & DONE & EPOS & GHEISHA & $\mathrm{x}$ & & $\mathrm{x}$ & $\mathrm{x}$ & DONE & $\begin{array}{l}70 \% \\
\text { DONE }\end{array}$ \\
\hline
\end{tabular}

Table 1(left): Summary of heavy nuclei induced showers produced. Currently only the production without contribution of Cherenkov radiation is completed. Table 2 (right): Summary of the proton-induced showers produced. The production with $\alpha=-2$ is completed while the flat spectrum production is ongoing

The production of each model is divided in 7 energy bins, equally logarithmically spaced from $10^{3} \mathrm{GeV}$ to $10^{9} \mathrm{GeV}$. The number of events in each energy range has been chosen according to the computing load (Table 3).

\begin{tabular}{|l|l|}
\hline Energy range $(\mathrm{GeV})$ & Number of events \\
\hline $200-100$ & 10000000 \\
\hline $10^{3}-10^{4}$ & 10000008 \\
\hline $10^{4}-10^{5}$ & 1000002 \\
\hline $10^{5}-10^{6}$ & 100000 \\
\hline $10^{6}-10^{7}$ & 10000 \\
\hline $10^{7}-10^{8}$ & 1000 \\
\hline $10^{8}-10^{9}$ & 100 \\
\hline
\end{tabular}

Table 3: Number of simulated events for each energy bin.

The computing time (fig. 1) and the size of the output files (fig. 2) increase with the energy of the primary cosmic ray.

Computation time per shower

loganithmic ally equally spaced bins with $E^{\wedge}-2$ energy spectrum

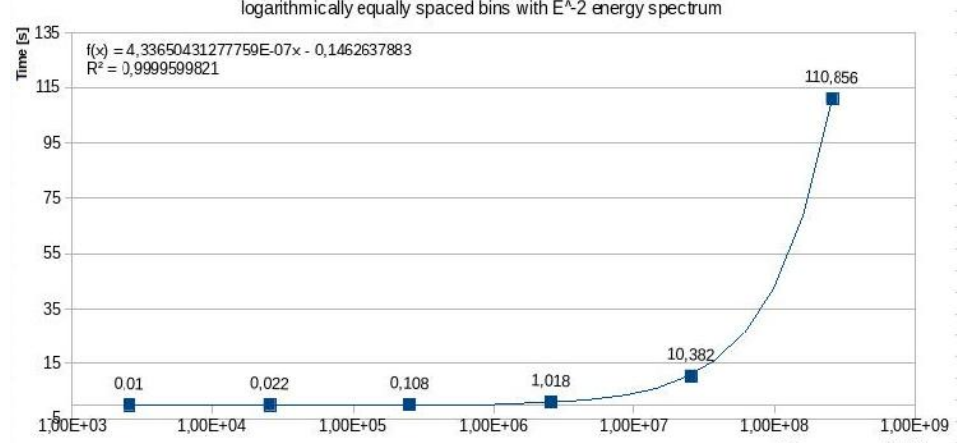

Figure 1: The computing time is the difference between the last and the first "PRESENT TIME" UTC date in the standard output of CORSIKA; the uncertainty in the computing time is of the order of 2 seconds.

All the information about every single shower is stored in a SQLite database that can be queried by the user. In order to simplify the access to the library, the information about secondary 
S.M.Stellacci, B.Spisso, C.Pellegrino, C.Bozza, T.Chiarusi, R.Coniglione, E.Giorgio, P.Lo Re, P.Migliozzi, A. Martini - CORELib

cosmic rays is extracted from CORSIKA output files and put in separated ASCII files (electromagnetic component, muons, neutrinos, hadrons+tau), but only for showers simulated without Cherekov effect.

We use a dedicated tool named CORANT, originally developed and maintained by T. Chiarusi, L. A. Fusco and C. Pellegrino and later improved by B. Spisso and S.M. Stellacci for this purpose.

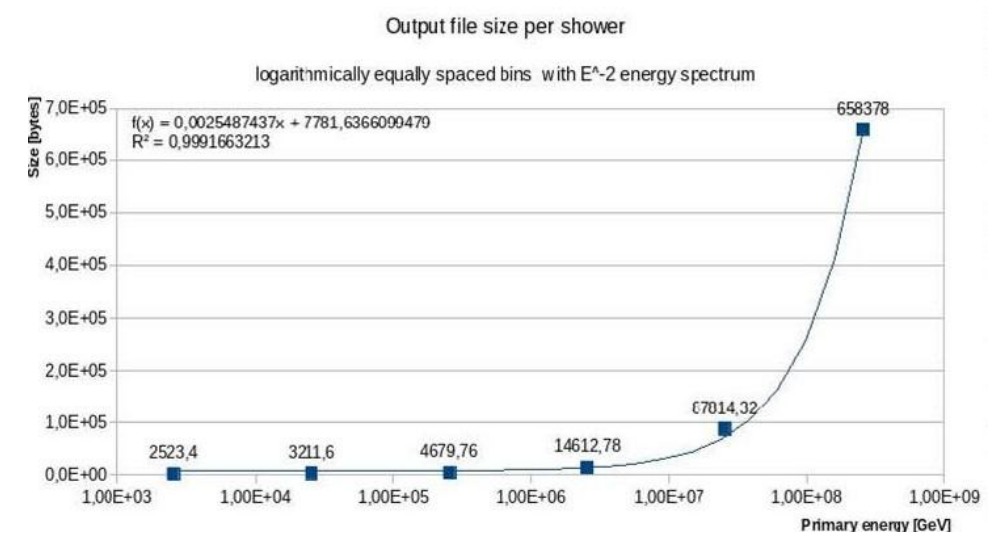

Figure 2: Average size in each energy bin.

The productions completed so far (March 2019) are stored at CNAF, the Information Technology National Center of INFN (Italian Institute for Nuclear Physics). They can be downloaded through gridFTP with an X509 certificate.

\section{CONCLUSIONS}

CORELib results from a big computation effort to feature large statistics of cosmic ray showers. Because of the wealth of simulated data, the productions can be used by a large community of researchers. The inherent flexibility of CORSIKA allows easy extension of the production to specific case, if requested. Last but not least, the dedicated access through X509 certificate is both open and secure

\section{ACKNOWLEDGEMENTS:}

We acknowledge the support of the European Commission Grant 653477 (ASTERICS) in the framework of the Horizon 2020-INFRADEV programme.

\section{References}

[1] D. Heck, J. Knapp, J.N. Capdevielle, G. Schatz, T. Thouw, "CORSIKA: A Monte Carlo Code to Simulate Extensive Air Showers", Forschungszentrum Karlsruhe Report FZKA 6019 (1998)

[2] Letter of Intent for KM3NeT 2.0, the KM3NET Collaboration, J.Phys. G 43084001 [arXiv:1601.07459] (2016) 